\title{
Focal atrophy of hypothalamus associated with third ventricle enlargement in Autism Spectrum Disorder
}

\author{
Farah Wolfe, Guillaume Auzias, Christine Deruelle, Thierry Chaminade* \\ Institut de Neurosciences de la Timone UMR 7289, Aix Marseille Université - \\ Centre National de la Recherche Scientifique, 13005 Marseille, France \\ *Corresponding author: \\ Tel:(+33) 491324032 . \\ E-mail: thierry.chaminade@univ-amu.fr
}

Word and Character Counts:

Abstract: 196

Total characters in body text (with spaces): 18384

Figures equivalent: 1625

The authors declare no conflict of interest. 
Hypothalamus and Autism 2

\section{ABSTRACT}

The hypothalamus is a brain structure containing multiple nuclei that mediate essential behavioral, autonomic and endocrinal functions including oxytocin (OXT) synthesis. OXT is a neuropeptide linked to complex social cognition and behaviors necessary for an effective social interaction. Oxytocinergic system dysfunction has been linked to social deficits in Autism Spectrum Disorders (ASD).

Limited studies have been done on the hypothalamus due to its small size and methodological constraints in current technology. This neuroimaging study examines hypothalamic atrophy in ASD in comparison to typically-developing (TD) population 1) by directly measuring grey matter (GM) density using a region-of-interest (ROI) analysis using Voxel-based morphometry (VBM), in a homogenous sample of participants controlled for age and IQ; 2) for generalization, by measuring third ventricular volume, based on its position bilaterally surrounded by the hypothalamus, using Freesurfer in a heterogeneous sample of participants.

A VBM analysis of CSF density on the first sample provides a link between GM density and third ventricle volume. Our results showed decreased hypothalamic GM density and increased third ventricle volume in ASD compared to TD subjects. Our findings provide neuroanatomical insights to social deficits in ASD that might be relevant for other psychiatric conditions.

Keywords: Hypothalamus, Autism, oxytocin, third ventricle, social deficit, neuroimaging 
Hypothalamus and Autism 3

\section{Introduction}

In the recent years, there has been a growing wealth of literature investigating pathophysiological mechanisms underlying social deficits in Autism Spectrum Disorders (ASD), a condition that affects 1 in 132 individuals [1]. Social deficit is one of the hallmark diagnostic criteria of ASD. However, understanding of its causes remains exploratory potentially due to the vast heterogeneity of genetic and phenotypic characteristics of the disorder. Recent research trends have pointed towards oxytocinergic dysfunction as a probable contributor to this deficit but its exact implications remain controversial [2]. Oxytocin (OXT) is considered a "social neuropeptide" synthesized in the hypothalamus involved in bonding, attachment and other complex social behaviors that are essential for social survival and effective conspecific interactions. OXT is produced and secreted from the paraventricular nucleus (PVN) and supraoptic nucleus (SON) of the hypothalamus where it subserves dual role, both as a hormone and neurotransmitter. It acts peripherally through the bloodstream as a hormone and centrally as a neurotransmitter, through axonal connections to other critical brain regions (eg., amygdala, striatum, nucleus accumbens, hippocampus etc.). In association with other neurohormones, OXT is responsible for mediating positive social emotions and behaviors [3], social memories [4] and motivation for social interaction [5].

The level of plasma OXT is abnormal in ASD and this abnormality is linked to dysregulation of oxytocin signaling pathways [6]. Studies have also proposed polymorphism of the OXT receptor as a genetic contribution to oxytocinergic dysfunction in ASD [7]. Furthermore, neuroimaging studies have demonstrated hypothalamic dysfunctions in ASD. For example, a study by Chaminade et al. [8] reported increased activation in the hypothalamus, putatively in the PVN, when typically-developing (TD), but not ASD participants, engaged in game playing with human 
versus robot and computer. This suggests that, in ASD, the lack of modulation by social context of the hypothalamus activity may be linked to OXT dysregulation. A neuroanatomical study by Kurth et al. [9] measured global brain size and tissue volume as well as regional grey matter (GM) difference of whole-brain MRIs of a large sample of participants using Voxel-Based Morphometry (VBM). They found atrophy in hypothalamic grey matter in ASD children compared to TD subjects, also putatively in regions corresponding to the SON and PVN of the hypothalamus. Thus, these findings indicate functional and anatomical differences in the hypothalamus of ASD that are postulated to reflect abnormal oxytocinergic modulatory mechanisms, plausibly from a reduction of positive feedback signal related to social motivation leading to a focal atrophy of the oxytocin-secreting regions (PVN and SON).

Through its multiple nuclei, the hypothalamus subserves many critical brain functions in coordinating endocrinal and autonomic activities that are important for human emotions and temper. Most findings on macrostructural abnormalities of the hypothalamus were based on postmortem studies thus providing a "retrospective perspective" [10], useful as a groundwork for MRI studies. Nonetheless, in vivo research of the hypothalamus is still imperative but the location and relatively small size of the hypothalamus present methodological challenges for MRI [11]. At present, technical evaluation of the anatomical boundaries of the hypothalamus in MRI research have been done using morphometric analysis to measure global hypothalamic volume [12], by mapping standardized structural delineations from MR scans of post-mortem anatomical sections [11] and most recently by creating a computer-assisted algorithm for segmentation in MRI [13].

Here, we hypothesized that, in ASD, there will be a hypothalamic atrophy associated with an increase in third ventricular volume, given its position is bilaterally surrounded by the 
hypothalamus. We first used a classical method using VBM in a homogeneous sample of participants controlled for age, gender and IQ. To explore a decrease in the hypothalamic grey matter density in ASD, we improved the previous study of Kurth and colleagues [9] by establishing a manually-segmented region of interest (ROI) a priori, to have a more precise localization. We found reduction in hypothalamic GM density with associated increase of cerebrospinal fluid (CSF) density corresponding to an enlargement of the third ventricle, within our ROI. Secondly, we tested the hypothesis of an increased third ventricle volume in ASD. Contrary to the tedious delineation of hypothalamic grey matter, the volume of the ventricles can be computed automatically using Freesurfer due to the high contrast between CSF and periventricular tissues in MRI. Measuring ventricular volume is thus more suited for systematic analysis on large cohorts. To increase generalizability of our results, we therefore included a larger and heterogeneous sample of participants. We included the lateral ventricle volume as a covariate so that the relative increase of volume in ASD can only be accounted by the properties of adjoining tissues and not by possible overall brain enlargement in ASD. By combining these two complementary methods, we provide a two-fold perspective in the evaluation of hypothalamic atrophy in ASD.

\section{Methods}

Direct approach

\section{Structural segmentation of ROI}

Segmentation was done on a high-resolution template image from the mni_colin27 template [14]. The template image is an average of 27 T1-weighted MRI scans of a single subject which was automatically transformed into the Talairach stereotaxic space. It resulted in a higher quality 
anatomical image with enhanced signal and finer resolution $(0.5 \mathrm{~mm}$ isotropic), which was crucial to maximize delineation accuracy in our manual segmentation of ROI.

A mask of the hypothalamus was created on the basis of published guidelines $[11,13]$ by four independent observers. The hypothalamus was outlined slice-by-slice starting on a coronal plane, tracing left and right hypothalami in separation. Manual segmentation began on the first slice where the continuous anterior commissure (AC) and optic chiasm was distinctly identified, moving anteriorly reaching the superior boundary of the fornix. The tuberal region was identified when the hypothalamus was at its widest, represented by the medial pole of the internal capsule, intersecting the third ventricle wall and extended to the superior border of the putamen. The posterior area was identified at the appearance of foramen of Monroe and hypothalamic sulcus which continued downward to the mammillary bodies, where the limit was set. The final slice preceded the first slice where the mammillary bodies and fornixes became prominent. The third ventricle was used to separate the right from the left hypothalamus. To obtain a binary mask for our ROI, we first selected voxels attributed to the hypothalamus by at least two observers and then applied a morphological closing of $2 \mathrm{~mm}$ to regularize the contour of the mask. The final ROI mask thus included voxels in the third ventricular space between the left and right hypothalamic GM.

Voxel-based morphometry (VBM)

Previously recorded sample of 20 male participants (10 ASD, 21.0 +/- 0.9, range $=18-25$ years old; 10 CTL, $21.9+/-0.6$, range $=19-25$ years old) without co-morbidities that were matched for age and verbal IQ were used in this study. Detailed demography can be found in the relevant publication [8]. An advanced VBM procedure using DARTEL registration [15] was used. First, 
three tissue classes resulting from segmentation, GM, CSF and white matter (WM) were used to calculate the diffeomorphic registration of the 20 subjects. Second, normalized images for the three tissue classes were resampled at $1 \mathrm{~mm}^{3}$ and smoothed with a $5 \mathrm{~mm}$ kernel. Finally, three independent-sample t-tests compared the density of the three tissue classes between the two groups within the hypothalamus mask to identify focal difference. The effects of age, IQ and global brain volume on the group difference were assessed using ANCOVA on tissue density extracted from clusters identified in the volumetric analysis. To control for the extent of multiple comparison, only voxels included in the ROI mask were considered in the model. A stringent threshold ( $\mathrm{p}<0.05$ family-wise error corrected) was used to control for type II error (minimum extent of 10 voxels).

Indirect approach

\section{Freesurfer}

To generalize findings to a less constrained population of ASD patients, a larger dataset $(n=76$, 41 ASD, $22.2+/-6.7$, range $=12-42$ years old; 35 CTR, $21.6+/-7.8$, range $=9-39$ years old) was obtained from $\mathrm{ABIDE}^{1}$ database, a MRI data-sharing platform that combines datasets of ASD and TD populations from 16 international sites which include phenotypic specifications for each subject. We selected a single site to ensure homogeneity of MRI quality within our large sample. USM site yielded the largest number of subjects that matched our inclusion criteria. We included only male subjects. Majority of the subject exclusion was due to other confounding conditions and the use of medication. Two subjects were excluded due to poor image resolution. We used Freesurfer automatic segmentation. This method provides an automatic segmentation on a

\footnotetext{
${ }^{1}$ http://fcon_1000.projects.nitrc.org/indi/abide/
} 
Hypothalamus and Autism 8

structural level and was shown to be comparable to manual delineation accuracy [16]. It allows for volumetric analysis of macroscopically visible brain structures by using a model that assign structural identity that is not solely restricted by spatial location, but also takes into account spatial relationships between anatomical structures. However, at present the range of anatomical structures that are registered in this tool does not include the hypothalamus, so were unable to investigate the hypothalamus in the analysis with this method. Instead, we identified ventricular volumes i.e., third ventricle, CSF (referring to the interventricular foramen between third and lateral ventricle), left, right and inferior parts of the lateral ventricle as well as choroid for the two groups (ASD, TD). We computed the volume of the third ventricle by summing up third ventricle and CSF and of the lateral ventricle by summing up left, right, inferior, lateral and choroid for each participant of the two groups (ASD, TD). Z-scores were computed on all of the measured variables in order to identify inaccurate segmentation and subjects with Z-value greater than \pm 3.0 were excluded.

\section{Ventricular volume}

In this second method, we analyzed the volumetric difference of the third ventricle between the two groups. It is known that cofactors such as global brain volume and age could significantly influence analysis and interpretation of significant effects [17]. Using global brain volume can be a confounding factor due to a possible brain enlargement in ASD [18], hence the effect of group on the volume of the third ventricle was analyzed using volume of the lateral ventricle as covariate. As the CSF is a continuous fluid throughout the ventricular spaces, relative differences between these two ventricular volumes can only be explained by the properties of surrounding tissues. 
Hypothalamus and Autism 9

A general linear model approach was used to analyze factors influencing the volume of the third ventricle. Group (ASD, CTL) was used as categorical variable, and Age as well as Volume of the lateral ventricles were used as continuous covariates. First-degree interaction between group and covariates were calculated. Analyses were performed using SPSS.

\section{Results}

Direct approach: VBM and ROI

The binary mask is presented in figures $1 \mathrm{a}$ and $1 \mathrm{~b}$. Second-level analysis of DARTEL processed and normalized grey matter (GM) tissue within the hypothalamus mask yielded a significant small cluster of increased density in CTL compared to ASD (x, y, z = 0, -1, -11; $\mathrm{P}_{\mathrm{FWE}}=.039$; $\mathrm{k}=31$ ). When rendered on the mean of all GM images, the cluster appeared to be located in the third ventricle (Figure 1c), so we performed second-level analysis of the CSF tissue within the hypothalamus mask. A highly significant cluster of increased CSF density in ASD compared to CTL (Figure 1d) was found adjacent to the GM cluster ( $\mathrm{x}, \mathrm{y}, \mathrm{z}=-1,-3,-4 ; \mathrm{P}_{\mathrm{FWE}}=.002 ; \mathrm{k}=119$ ). According to the MNI coordinates in the hypothalamus atlas [11], these two clusters are closest to the paraventricular nucleus of the anterior hypothalamic area. No focal difference between groups was found for the WM density at the stringent thresholds used.

Respective raw GM and CSF density in reported clusters were extracted for each participant and further investigated to verify the effect of age, IQ and global brain volume on the group difference. In all three ANCOVAs assessing the main effect of group and each of the covariates, the former was always significant $($ all Fs $(1,20)>10.90, p s<0.005)$ and the later never reached significance (all Fs $(1,20)<2.5, p s>0.15)$. 
Indirect approach: Ventricular volume

ANCOVA indicated that the third ventricle was significantly influenced by diagnosis $(\mathrm{F}(1,67)=6.71, p=.01)$ as well as by the volume of the lateral ventricles $(\mathrm{F}(1,67)=7.37$, $p=.01$; see figure). The effect of age $(\mathrm{F}(1,67)=0.57, p=0.45)$ and interactions between diagnosis and age on the one hand $(\mathrm{F}(1,67)=1.75, p=.19)$, diagnosis and volume of lateral ventricle on the other hand $(\mathrm{F}(1,67)=1.69, p=.20)$, did not reach significance. There was a highly significant $(p<0.001)$ increase of third ventricle volume (residuals after regressing out covariates, i.e. age and lateral ventricle volume) in ASD compared to CTL.

$\sim$ Figure 2 around here

\section{Discussion}

In this study, we analyzed hypothalamic GM density and third ventricular volume in ASD in comparison to TD population, using two complementary approaches. We postulated that hypothalamic atrophy would be reflected in the volume of adjoining structure, in this case, the third ventricle. Our results revealed a cluster of significant decrease in hypothalamic GM in ASD congruent to previous finding [9]. In its vicinity, we found a cluster of increased CSF density within the third ventricle close to the PVN within our ROI mask, leading to a volumetric analysis of the third ventricle. Finally we report an increase of third ventricle volume independent of the lateral ventricles volume in ASD [19], in agreement with prior reports and our hypothesis.

We used the VBM method to reproduce the finding of a focal reduction of grey matter density in the hypothalamus. To control for intersubject brain variability accounted by extraneous factors such as age, gender and IQ, a tightly matched homogenous groups of participants was used. Manual segmentation and high spatial resolution were required to increase precision in the 
Hypothalamus and Autism 11

attribution of focal changes to the hypothalamic nuclei in our ROI analysis, thus we utilized this method to measure focal hypothalamic GM difference using small voxel size of $1 \mathrm{~mm}^{3}$. We report decreased GM density for ASD in a cluster closest to the PVN area. The PVN contains neurosecretory neurons that centrally project neuropeptides including OXT to other brain regions, hence abnormalities in this hypothalamic nucleus provide support for the hypothesis of oxytocin dysfunction in ASD.

Our analysis also supports the simultaneous presence of an enlargement of the third ventricle in response to the hypothalamic atrophy in ASD. As mentioned in the methods, our binary mask included the hypothalamus as well as the third ventricle. When three tissue classes were investigated, we found increased CSF and decreased GM density co-localized in the third ventricle for ASD when compared to TD in the absence of significant WM change. This result suggests that the grey matter is replaced by ventricular space in ASD, hence a hypothalamic atrophy.

To validate the reproducibility of hypothalamic atrophy in ASD, we analyzed the third ventricular volume using Freesurfer tool on a large, heterogeneous sample. This method served as an indirect assessment because only the ventricles could be analyzed. Nonetheless, it was useful because it is automatic, hence easily applicable to generalize the finding of third ventricle enlargement to a large sample of participants. Total brain volume increase, consequential to brain enlargement, has been observed in ASD [18]. To control for this possibility, the lateral ventricle was used as a covariate instead of global brain volume under the assumption that as the CSF is one continuous fluid with constant pressure throughout, relative differences between these two volumes can only be explained by the properties of adjoining tissues. Moreover, research on ventricular volume in ASD population have concluded with mixed findings with 
some demonstrated an increase and some a decrease [20] but only one study showed an increase in third ventricle [19]. Our results revealed significant increase of third ventricular volume in ASD independent of the lateral ventricle volume, suggesting that this enlargement occurs independent of the potential megalencephaly in this population.

Interestingly, similar ventricular abnormalities have also been found in other disorders. A study of bipolar patients showed increase in third ventricle volume, which was postulated to be associated with dysfunctions of hypothalamic nuclei [21]. Our results therefore support that enlarged third ventricle may be a result of physical adjustment consequential to focal atrophy of the hypothalamus over the span of neurodevelopment.

\section{Conclusion}

The hypothalamus is proven pertinent in numerous socially-relevant emotional, behavioral and physiological processing and these aspects were shown to be impaired in ASD. Its multiple nuclei serve different purposes and project to various neural networks. Hence, atrophy of the hypothalamus could be related to neurodevelopmental feedback loop, possibly associated with oxytocinergic system dysfunction in ASD. Using an animal model it was shown that 4 weeks of social isolation resulted in increase not only in plasma level of circulating oxytocin, but also of oxytocin-immunoresponsive cells in the PVN, illustrating the plasticity of the hypothalamus at the cytoarchitectonic level [22]. Future investigations that will allow for assessment at various

Commented [T4]: Added neurodevelopmental stages, perhaps in a form of longitudinal study commencing in the early years, are warranted to further understand the pathophysiological basis of this atrophy in ASD. the hypothalamus, is another common dysfunction found in ASD [23] and a wide range of other 
psychiatric disorders [24]. In addition, hypothalamic atrophy was also found in patients with anxiety disorder [25] and schizophrenia [26], both of which are conditions involving social impairments. of prairie voles

Thus supporting our results, these pieces of evidence suggest that hypothalamic atrophy may have substantial impact on various neurophysiological functions and phenotypic expressions relating to social deficits, which could shed some light to symptomatological commonalities that exist in ASD as well as several other psychiatric disorders. 


\section{References}

1. Baxter AJ, Brugha TS, Erskine HE, Scheurer RW, Vos T, Scott JG. The epidemiology and global burden of autism spectrum disorders. Psychol Med. 2014; 1-13

2. Zik JB \& Roberts D. The many faces of oxytocin: Implications for psychiatry. Psychiatry Research. 2015; 226:31-37

3. Uvnäs-Moberg K. Oxytocin may mediate the benefits of positive social interaction and emotions. Psychoneuroendocrinology. 1998; 23: 819-835

4. Guastella AJ, Mitchell PB, Frosso M. Oxytocin Enhances the Encoding of Positive Social Memories in Humans. Biol Psychiatry. 2008; 64: 256-258

5. Chevallier C., Kohls G., Troiani V, Brodkin ES, Schultz RT. The social motivation theory of autism. Trends in Cog Sciences. 2012; 16(4) :231-239

6. Jacobson JD, Ellerbeck KA, Kelly KA, Fleming KK, Jamison TR et al. Evidence for alterations in stimulatory $\mathrm{G}$ proteins and oxytocin levels in children with autism Psychoneuroendocrinology. 2014;40:159-169

7. Freitag CM, Wouter S, Klauck SM, Duketis E, Waltes R. Genetics of autistic disorders: review and clinical implications. European Child \& Adolescent Psychiatry. 2010; 19: 169178

8. Chaminade T, Da Fonseca D, Rosset D, Cheng G, Deruelle, C. Atypical modulation of hypothalamic activity by social context in ASD. Res in ASD. 2015; 10:41-50

9. Kurth F, Narr KL, Roger PW, O’Neill J, Alger JR et al. Diminished grey matter within the hypothalamus in autism disorder: a potential link to hormonal effects? 2011; 70 (3): 278-282

10. Schindler S, Geyer S, Straus M, Anwander A, Hegerl U et al. Structural studies of the hypothalamus and its nuclei in mood disorders. Psychiatry Research: Neuroimaging. 2012; 201: 1-9

11. Baroncini M, Jissendi P, Balland E, Besson P, Pruvo JP et al. MRI atlas of the human hypothalamus. Neuroimage. 2012; 59, 168-180

12. Goldstein JM, Seidman LJ, Makris N, Ahem T, O'Brien LM, et al. Hypothalamic abnormalities in schizophrenia: Sex effects and genetic vulnerability. Biol Psychiatry. 2007; 61: 935-945

13. Schindler S, Schönknecht P, Schmidt L, Anwander A, Straus M et al. Development and Evaluation of an Algorithm for the Computer-Assisted Segmentation of the Human 
Hypothalamus and Autism 15

Hypothalamus on 7-Tesla Magnetic Resonance Images. PLoS ONE. 2013; 8(7)

14. Holmes CJ, Hoge R, Collins L, Woods R, Toga AW et al. Enhancement of MR images using registration for signal averaging. J Comput Assist Tomogr.1998;22(2):324-33. http://dx.doi.org/10.1097/00004728-199803000-00032

15. Ashburner J. A fast diffeomorphic image registration algorithm. Neuroimage.2007;38(1):95113.

16. Fischl B, Salat DH, Busa E, Albert M, Dieterich M et al. Whole brain segmentation: automated labeling of neuroanatomical structures in the human brain. Neuron. 2002;33:341355

17. Aylward EH, Minshew NJ, Field K, Sparks BF, Singh N. Effects of age on brain volume and head circumference in autism. Neurology. 2002; 59:175-183

18. Stanfield AC, McIntosh AM, Spencer MD, Philip R, Gaur S et al. Towards a neuroanatomy of autism: A systematic review and meta-analysis of structural magnetic resonance imaging studies. Eur Psychiatry. 2008;23:289 -299

19. Hardan AY, Minshew NJ, Mallikarjuhn M, Keshavan MS. Brain volume in autism. J Child Neurol. 2001;16:421-424

20. Brambilla P, Hardan A, Ucelli di Nemi S, Perez J, Soares JC et al. Brain anatomy and development in autism: review of structural MRI studies. Brain Research Bulletin. 2003; 61: 557-569

21. Bhadoria R, Watson D, Danson P, Ferrier IA, Mcallister V et al. Enlargement of the third ventricle in affective disorders. Ind J Psychiatry. 2003; 45:147-150.

22. Grippo, A. J., Gerena, D., Huang, J., Kumar, N., Shah, M., Ughreja, R., \& Carter, C. S. (2007). Social isolation induces behavioral and neuroendocrine disturbances relevant to depression in female and male prairie voles. Psychoneuroendocrino. 2007. 32:966-980

23. Tordjman S, Davlantis K, Georgieff N, Geoffray M, Speranza M et al. Autism as a disorder of biological and behavioral rhythms: towards new therapeutic perspectives. Front Pediatr. $2015 ; 3: 1$

24. Boivin DB. Influence of sleep-wake and circadian rhythm disturbances in psychiatric disorders. J Psychiatry Neurosci. 2000;25:446-458

25. Terlevic R, Isola M, Ragogna M, Meduri M, Canalaz F et al. Decreased hypothalamus volumes in generalized anxiety disorder but not in panic disorder. J Affect Disorder. 2013; 
Hypothalamus and Autism 16

146: 390-394

26. Koolschijn PC, van Haren N, Hulshoff Pol HE, Kahn RS. Hypothalamus volume in twin pairs discordant for schizophrenia. European Neuropsychopharmacology. 2008; 18: 312-315 
Figures and Legends
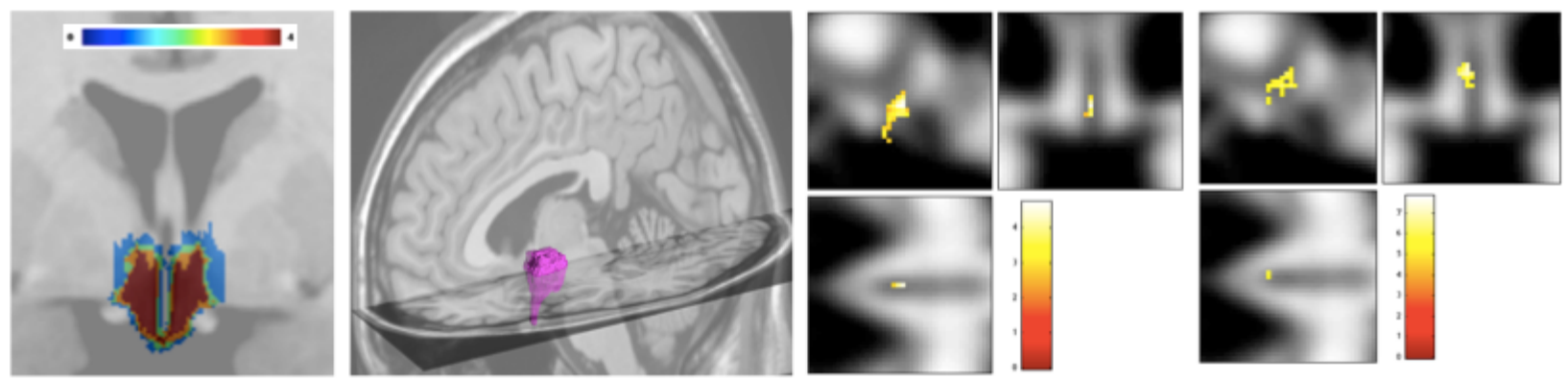

Figure 1. a) Template of the ROI mask showing inter-rater delineation; voxels by at least 2 observers were included. b) 3D render of the final binary mask. c) Increase in GM density in CTL compared to ASD. d) Increase in CSF density in ASD compared to CTL. In (c) and (d) renders are on the mean across all GM images of participants.

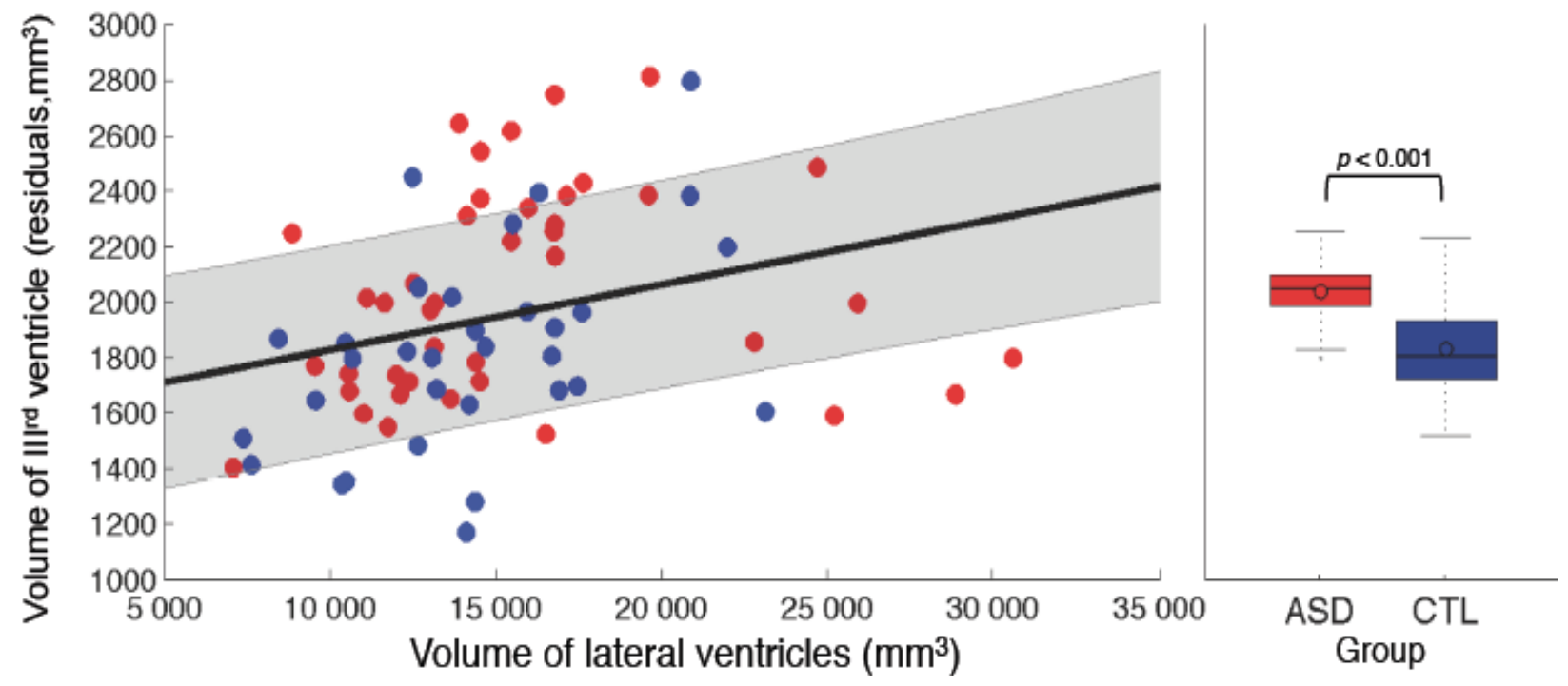

Figure 2: The volume of the third ventricle is significantly influenced by the volume of the lateral ventricles (left) as well as the group, with an enlargement for individuals diagnosed with ASD. 